\section{A FIRST-IN-HUMAN PHASE I DOSE-ESCALATION TRIAL OF THE B7-H6/CD3 T-CELL ENGAGER BI $765049 \pm$ EZABENLIMAB (BI 754091) IN PATIENTS WITH ADVANCED SOLID TUMORS EXPRESSING B7-H6}

${ }^{1}$ Gerald Falchook* ${ }^{2}$ David Spigel, ${ }^{3}$ Manish Patel, ${ }^{4}$ Babar Bashir, ${ }^{5}$ Susanna Ulahannan, ${ }^{6}$ Christine Duffy, ${ }^{7}$ Daniela Maier, ${ }^{8}$ Hisaya Azuma. ${ }^{1}$ Sarah Cannon Research Institute at HealthONE, Denver, CO, USA; ${ }^{2}$ Sarah Cannon Research Institute/Tennessee Oncology, PLLC, Nashville, TN, USA; ${ }^{3}$ Florida Cancer Specialists and Sarah Cannon Research Institute, Sarasota, FL, USA; ${ }^{4}$ Sidney Kimmel Cancer Center at Thomas Jefferson University, Philadelphia, PA and Sarah Cannon Research Institute, Philadelphia, PA, USA; ${ }^{5}$ The University of Oklahoma Health Sciences Center/Sarah Cannon Research Institute, OKC, OK, USA; ${ }^{6}$ Boehringer Ingelheim Pharmaceuticals Inc., Ridgefield, CT, USA; ${ }^{7}$ Boehringer Ingelheim Pharma GmbH and Co. KG, Biberach/Riss, Germany; ${ }^{8}$ Boehringer Ingelheim International GmbH, Ingelheim am Rhein, Germany

Background B7-H6 is a member of the B7 family of immune receptors, which is expressed in several solid tumor types but very little expression can be detected in normal tissues. ${ }^{12}$ BI 765049 is a novel IgG-like bispecific T-cell engager designed to bind simultaneously to B7-H6 on tumor cells and $\mathrm{CD} 3$ on $\mathrm{T}$ cells, resulting in cytolytic synapse formation and tumor lysis. Preclinical studies have demonstrated that BI 765049 monotherapy induced dose-dependent anti-tumor activity in humanized in vivo CRC tumor models. Consistent with the mode of action, the treatment with BI 765049 led to target cell apoptosis, local T-cell activation/proliferation and cytokine production in the tumor tissue, with $\mathrm{PD}-(\mathrm{L}) 1$ upregulation. ${ }^{3}$ Activation of the $\mathrm{PD}-(\mathrm{L}) 1$ provides the rationale for combining BI 765049 with a PD1 inhibitor.

Methods NCT04752215 is a first-in-human, open-label, doseescalation trial of BI $765049 \pm$ the PD-1 inhibitor, ezabenlimab. Adults with advanced, unresectable and/or metastatic CRC, NSCLC, HNSCC, hepatocellular, gastric or pancreatic carcinoma are eligible. Patients must have failed on, or be ineligible, for standard therapies. B7-H6 positivity must be confirmed at screening by central review (immunohistochemistry assay) in archived tissues/in-study fresh biopsies (except CRC). Patients must have $\geq 1$ evaluable lesion (modified RECIST 1.1) outside of the central nervous system and adequate organ function. The primary objective is to determine the maximum tolerated dose (MTD) or recommended dose for expansion of BI $765049 \pm$ ezabenlimab, based on dose-limiting toxicities during the MTD evaluation period. Further objectives are to evaluate safety, tolerability, PK/PD and preliminary efficacy of BI $765049 \pm$ ezabenlimab. The trial may assess up to 4 dosing regimens: A (BI 765049 once every 3 weeks [q3w]); B1 (BI 765049 qw); B2 (BI 765049 qw with step-in doses); C (BI $765049+$ ezabenlimab [q3w]). Dose escalation will be guided by a Bayesian Logistic Regression Model with overdose control that will be fitted to binary toxicity outcomes using a hierarchical modelling approach to jointly model all dosing regimens. Treatment will be allowed to continue until confirmed progressive disease, unacceptable toxicity, other withdrawal criteria or for a maximum duration of 36 months, whichever occurs first. Approximately 150-175 patients will be screened and $\sim 120$ patients enrolled. As of July 2021, patients are being recruited in early dose-escalation cohorts.

Acknowledgements Medical writing support for the development of this abstract, under the direction of the authors, was provided by Becky O'Connor, of Ashfield MedComms, an Ashfield Health company, and funded by Boehringer Ingelheim.

Trial Registration NCT04752215

\section{REFERENCES}

1. Brandt et al. J Exp Med 2009;206:1495-503.

2. Boehringer Ingelheim. Data on file.

3. Hipp et al. AACR Annual Meeting 2021.

Ethics Approval The trial will be carried out in compliance with the protocol, the ethical principles laid down in the Declaration of Helsinki, in accordance with the ICH Harmonized Guideline for Good Clinical Practice (GCP) and the EU directive 2001/20/EC/EU regulation 536/2014.

http://dx.doi.org/10.1136/jitc-2021-SITC2021.480 